\title{
New conservation laws for inviscid Burgers equation
}

\author{
IGOR LEITE FREIRE \\ Centro de Matemática, Computação e Cognição, Universidade Federal do ABC - UFABC \\ Rua Santa Adélia, 166, Bairro Bangu, 09210-170 Santo André, SP, Brasil \\ E-mails: igor.freire@ufabc.edu.br / igor.leite.freire@gmail.com
}

\begin{abstract}
In this paper it is shown that the inviscid Burgers equation is nonlinearly self-adjoint. Then, from Ibragimov's theorem on conservation laws, local conserved quantities are obtained.

Mathematical subject classification: Primary: 76M60; Secondary: 58J70.

Key words: Lie point symmetry, Ibragimov's Theorem, conservation laws, inviscid Burgers equation, nonlinear self-adjoint equations, weak self-adjoint equations.
\end{abstract}

\section{Introduction}

In a previous work [4] the class of the self-adjoint equations (in the sense of the definition introduced by Ibragimov in [12]) of the type

$$
u_{t}+f\left(t, x, u, u_{x}\right)=0
$$

was determined. In particular, it was proved that the inviscid Burgers equation

$$
u_{t}+a(u) u_{x}=0
$$

is (quasi) self-adjoint. In addition, from Ibragimov's theorem on conservation laws [13], conservation laws for projectable Lie point symmetries (see [21]) of (1) were established.

Recently Maria Luz Gandarias [8] and Nail Ibragimov [17, 18, 19] have generalized the previous concepts of self-adjoint equations $[12,13,14]$.

\#CAM-701/12. Received: 22/IX/12. Accepted: 28/IX/12. 
The recent developments allow one to find new conservation laws for equation (1). Thus in this paper the results obtained in [4] are complemented by using the new concepts $[8,17,18,19]$ combined with the powerful result [13].

The paper is organized as the follows: in the next section it is revisited Ibragimov's theorem on conservation laws and the concepts of self-adjoint equations. In the Section 3 the results regarding (1) obtained in [4] are discussed with the point of view of the new developments. In the following, some new conservation laws are established illustrating the results.

\section{Revisiting previous results}

\subsection{Ibragimov's theorem on conservation laws}

In what follows, it is assumed the summation over repeated indices, $x=$ $\left(x^{1}, \ldots, x^{n}\right)$ and $u=\left(u^{1}, \ldots, u^{m}\right)$ denotes the indepedent variables and the depedents variables, respectively. The set of $k t h$ order derivatives and all differential functions of finite order shall be denoted by $\partial^{k} u$ and $\mathcal{A}$, respectively.

Ibragimov's theorem on conservation laws is

Theorem 2.1. Any symmetry (Lie point, Lie-Bäcklund, nonlocal symmetry)

$$
X=\xi^{i} \frac{\partial}{\partial x^{i}}+\eta^{\alpha} \frac{\partial}{\partial u^{\alpha}}+\eta_{i}^{\alpha} \frac{\partial}{\partial u_{i}^{\alpha}}+\eta_{i j}^{\alpha} \frac{\partial}{\partial u_{i j}^{\alpha}}+\cdots,
$$

where $\xi^{i}, \eta^{\alpha} \in \mathcal{A}, \eta_{i}^{\alpha}=D_{i}\left(\eta^{\alpha}-\xi^{j} u_{j}^{\alpha}\right)+\xi^{j} u_{i j}^{\alpha}, \eta_{i j}^{\alpha}=D_{i} D_{j}\left(\eta^{\alpha}-\xi^{k} u_{k}^{\alpha}\right)+$ $\xi^{k} u_{k i j}^{\alpha}$, etc, of the system of equations

$$
F_{\alpha}\left(x, u, \partial u, \ldots, \partial^{s} u\right)=0
$$

with $n$ independent variables $x=\left(x^{1}, \ldots, x^{n}\right)$ and $m$ dependent variables $u=\left(u^{1}, \ldots, u^{m}\right)$ is inherited by the adjoint equation. Specifically the operator

$$
Y=\xi^{i} \frac{\partial}{\partial x^{i}}+\eta^{\alpha} \frac{\partial}{\partial u^{\alpha}}+\eta_{*}^{\beta} \frac{\partial}{\partial v^{\beta}}
$$

with an appropriately chosen coefficient $\eta_{*}$ is admitted by the system of equations (3) and its adjoint system

$$
F_{\alpha}^{*}\left(x, u, v, \partial u, \partial v, \ldots, \partial^{s} u, \partial^{s} v\right):=\frac{\delta\left(v^{\beta} F_{\beta}\right)}{\delta u^{\alpha}}=0 .
$$


Furthermore, the combined system (3) and (5) has the conservation law $D_{i} C^{i}=0$, where

$$
\begin{aligned}
C^{i}= & \xi^{i} \mathcal{L}+W_{\alpha}\left[\frac{\partial \mathcal{L}}{\partial u_{i}^{\alpha}}-D_{j}\left(\frac{\partial \mathcal{L}}{\partial u_{i j}^{\alpha}}\right)+D_{j} D_{k} \frac{\partial \mathcal{L}}{\partial u_{i j k}^{\alpha}}-\cdots\right] \\
& +D_{j}\left(W^{\alpha}\right)\left[\frac{\partial \mathcal{L}}{\partial u_{i j}^{\alpha}}-D_{k}\left(\frac{\partial \mathcal{L}}{\partial u_{i j k}^{\alpha}}\right)+\cdots\right] \\
& +D_{j} D_{k}\left(W^{\alpha}\right)\left[\frac{\partial \mathcal{L}}{\partial u_{i j k}^{\alpha}}-\cdots\right]+\cdots
\end{aligned}
$$

and $W^{\alpha}=\eta^{\alpha}-\xi^{i} u_{i}^{\alpha}$.

\subsection{Quasi-self-adjoint and self-adjoint equations}

The following definitions were introduced in $[12,13,14]$.

Definition 2.2. An equation $F=0$ is said to be self-adjoint if there exists a function $\phi=\phi(x, u, \ldots)$ such that $\left.F^{*}\right|_{v=u}=\phi F$. Thus $\left.F^{*}\right|_{v=u}=0$ if and only if $F=0$.

Definition 2.3. An equation $F=0$ is said to be quasi-self-adjoint if there exists a function $\phi=\phi(x, u, \ldots)$ such that $\left.F^{*}\right|_{v=\varphi(u)}=\phi F$, with $\varphi^{\prime}(u) \neq 0$. Thus $\left.F^{*}\right|_{v=\varphi(u)}=0$ if and only if $F=0$.

Whenever an equation is quasi-self-adjoint or self-adjoint, formulae (6) allow one to construct local conservation laws for the considered equation insteading of $v$ by $\varphi(u)$ or $u$, respectively.

Recently many authors have been employing these concepts in order to establish conservation laws for equations and systems. For instance, Ibragimov, Torrisi and Tracinà determined the class of quasi-self-adjoint system derived from a $(2+1)$ generalized Burgers equation in [16]. Bruzón, Gandarias and Ibragimov determined a class of self-adjoint differential equations in [3]. Conservation laws for the Camassa-Holm equation was obtained by Ibragimov, Khamitova and Valenti in [20]. Further examples can be found in [4, 5, 15]. 


\subsection{Conservation laws for inviscid Burgers equation}

The adjoint equation to (1) is (see [4])

$$
v_{t}+a(u) v_{x}=0 .
$$

Let $F=v_{t}+a(u) u_{x}$. Then it is easy to see that $\left.F^{*}\right|_{v=\varphi(u)}=-\varphi^{\prime}(u)\left(u_{t}+\right.$ $\left.a(u) u_{x}\right)$. Thus (1) is quasi-self-adjoint, for all smooth function $\varphi=\varphi(u)$. In particular, this holds for $\varphi=u$ and equation (1) is also self-adjoint.

From Ibragimov's theorem on conservation laws, a conserved vector for equation (1) is

$$
\begin{aligned}
& C^{0}=\left[\eta+(\tau a(u)-\xi) u_{x}\right] \varphi(u), \\
& C^{1}=\left[\eta a(u)-(\tau a(u)-\xi) u_{t}\right] \varphi(u),
\end{aligned}
$$

where

$$
X=\tau(x, t, u) \frac{\partial}{\partial t}+\xi(x, t, u) \frac{\partial}{\partial x}+\eta(x, t, u) \frac{\partial}{\partial u}
$$

is a Lie point symmetry generator of (1).

\section{New conservation laws for equation (1)}

Definitions 1 and 2 have been extended to

Definition 3.1. An equation $F=0$ is said to be nonlinearly self-adjoint if the equation obtained from the adjoint equation (5) by the substitution $v=\varphi(x, u)$ with a certain function $\varphi(x, u) \neq 0$ is identical with the original equation (3), that is,

$$
\left.F^{*}\right|_{v=\varphi(x, u)}=\phi(x, u, \ldots) F,
$$

for some $\phi \in \mathcal{A}$.

Whenever (9) holds for a certain function $\varphi$ such that $\varphi_{u} \neq 0$ and $\varphi_{x^{i}} \neq 0$, the equation $F=0$ is called weak self-adjoint.

Remark 3.1. The concept of nonlinearly self-adjoint equations was introduced by Ibragimov, see $[17,18,19]$. On the other hand, the notion of weak self-adjoint equation was introduced by Gandarias in [8]. 
With respect to these new concepts, weak self-adjointness for evolution equations were obtained in $[9,10,11]$. Namely, in [10] Gandarias, Redondo and Bruzón applied the new concept to a class of equations arising in financial mathematics. In [9] Gandarias established local conservation laws for a porous medium equation using the self-adjointness of the equation under consideration. More recently Gandarias and Bruzón [11] found a class of weak self-adjoint forced $\mathrm{KdV}$ equations.

Nonlinearly self-adjointness have been focused by Freire and Sampaio in [6], where the authors determined a class of nonlinear self-adjoint equations of fifth-order. In [2] Bozhkov, Freire and Ibragimov showed that the nonlinear self-adjointness of the Novikov equation (further details, see [2] and references therein) implies in the strictly self-adjointness of that equation. In [7] new classes of self-adjoint equations up to fifth-order were found.

Taking the nonlinearly self-adjointness for differential equations into account, substituting $v=\psi(x, t, u)$ into (7) and using (1), it is obtained

$$
\psi_{t}+a(u) \psi_{x}=0
$$

A solution to (10) is $\psi(x, t, u)=\phi(z)+\varphi(u)$, with $z=x-t a(u)$.

From Ibragimov's theorem on conservation laws, a local conservation law for equation (1) is given by

$$
\begin{aligned}
& C^{0}=\left[\eta+(\tau a(u)-\xi) u_{x}\right](\phi(z)+\varphi(u)), \\
& C^{1}=\left[\eta-(\tau a(u)-\xi) u_{t}\right](\phi(z)+\varphi(u)),
\end{aligned}
$$

where $z=x-t a(u)$.

In fact, the new conserved vector obtained from the self-adjointness' new concept is $C=\left(C^{0}, C^{1}\right)$, where

$$
\begin{aligned}
& C^{0}=\left[\eta+(\tau a(u)-\xi) u_{x}\right] \phi(x-t a(u)), \\
& C^{1}=\left[\eta a(u)-(\tau a(u)-\xi) u_{t}\right] \phi(x-t a(u)) .
\end{aligned}
$$

Observe that (12) possibilites to find an infinite number of new conservation laws for a fixed Lie point symmetry generator of equation (1). 


\section{Examples}

Here it will be established some conservation laws illustrating the results obtained previously. Consider the Lie point symmetry generator

$$
X_{4}=t \frac{\partial}{\partial t}-\frac{a(u)}{a^{\prime}(u)} \frac{\partial}{\partial u}
$$

of equation (1). Here it is used the same notation employed in [4]. Substituting the components of (13) into (12) it is obtained

$$
\begin{aligned}
& C^{0}=\left[-\frac{a(u)}{a^{\prime}(u)}+\operatorname{ta}(u) u_{x}\right] \phi(x-t a(u)), \\
& C^{1}=\left[-\frac{a(u)^{2}}{a^{\prime}(u)}-t a(u) u_{t}\right] \phi(x-\operatorname{ta}(u)) .
\end{aligned}
$$

Setting $\phi(z)=z$ into (14), it is found that the conserved vector is $C=$ $\left(C^{0}, C^{1}\right)$, where

$$
\begin{aligned}
& C^{0}=-\frac{a(u)}{a^{\prime}(u)}(x-t a(u))-t A(u)+D_{x}\left(t x A(u)-t^{2} \alpha(u)\right), \\
& C^{1}=-\frac{a(u)^{2}}{a^{\prime}(u)}(x-t a(u))+x A(u)-2 t \alpha(u)+D_{t}\left(t^{2} \alpha(u)-t x A(u)\right)
\end{aligned}
$$

$\alpha$ is given by $A^{\prime}(u)=a(u)$ and $\beta$ is a function such that $\alpha^{\prime}(u)=a(u)^{2}$. Transfering the terms $D_{x}(\ldots)$ from $C^{0}$ to $C^{1}$ and simplifying, it is obtained the conserved vector $C=\left(C^{0}, C^{1}\right)$, with components given by

$$
\begin{aligned}
C^{0} & =-\frac{a(u)}{a^{\prime}(u)}(x-\operatorname{ta}(u))-t A(u), \\
C^{1} & =-\frac{a(u)^{2}}{a^{\prime}(u)}(x-t a(u))+x A(u)-2 t \alpha(u) .
\end{aligned}
$$

Recently new Lie point symmetries of the inviscid Burgers equations were found, see [1]. In the next example we use the new generator (it is employed the same notation of the original paper [1])

$$
Z_{11}=(x-t a(u)) \frac{\partial}{\partial x}
$$

for establishing another conservation laws for (1). Let $\phi=c_{1}(x-\operatorname{ta}(u))+c_{2}$, $\varphi=c_{3} u^{p}+c_{4} / u, p \neq-1$, where $c_{1}, c_{2}, c_{3}, c_{4} \in \mathbb{R}$ are arbitrary constants, 
$A^{\prime}(u)=a(u), B^{\prime}(u)=a(u) u^{p}, U^{\prime}=a(u) / u$ and $\alpha^{\prime}=a(u)^{2}$. From (10) it is obtained

$$
\begin{aligned}
C^{0}= & -c_{1}(x-\operatorname{ta}(u))^{2} u_{x}-c_{2}(x-\operatorname{ta}(u)) u_{x} \\
& -c_{3}(x-\operatorname{ta}(u)) u_{x}-c_{4} \frac{x-\operatorname{ta}(u)}{u} u_{x}, \\
C^{1}= & c_{1}(x-\operatorname{ta}(u))^{2} u_{t}+c_{2}(x-\operatorname{ta}(u)) u_{t} \\
& +c_{3}(x-\operatorname{ta}(u)) u_{t}+c_{4} \frac{x-\operatorname{ta}(u)}{u} u_{t} .
\end{aligned}
$$

Since

$$
\begin{aligned}
C^{0}= & c_{1}\left[D_{x}\left(2 x t A-x^{2} u-t^{2} \alpha\right)+2 x u-2 t A\right]+c_{2}\left[D_{x}(t A-x u)+u\right] \\
& +c_{3}\left[D_{x}\left(t B-x \frac{u^{p+1}}{p+1}\right)+\frac{u^{p+1}}{p+1}\right]+c_{4}\left[D_{x}\left(x \ln |u|+\frac{t a(u)}{u}\right)+\ln |u|\right], \\
C^{1}= & c_{1}\left[D_{t}\left(x^{2} u+t^{2} \alpha-2 x t A\right)+2 x A-2 t \alpha\right]+c_{2}\left[D_{t}(x u-t A)+A\right] \\
& +c_{3}\left[D_{t}\left(x \frac{u^{p+1}}{p+1}-t B\right)+B\right]+c_{4}\left[D_{t}(x \ln |u|-t U)+U\right],
\end{aligned}
$$

by transfering the terms $D_{t}(\ldots)$ from $C^{1}$ to $C^{0}$, it is obtained

$$
\begin{aligned}
C^{0} & =c_{1}(2 x u-2 t A)+c_{2} u+c_{3} \frac{u^{p+1}}{p+1}+c_{4} \ln |u|, \\
C^{1} & =c_{1}(2 x A-2 t \alpha)+c_{2} A+c_{3} B+c_{4} U .
\end{aligned}
$$

It is easy to see that the conserved vector obtained is a linear combination of the conserved vectors

$$
\begin{aligned}
& D_{1}=(2 x u-2 t A, 2 x A-2 t \alpha), \quad D_{2}=(u, A), \\
& D_{3}=\left(\frac{u^{p+1}}{p+1}, B\right), \quad D_{4}=(\ln |u|, U) .
\end{aligned}
$$

\section{Conclusion}

In this paper the previous results on conservation laws obtained by the author in [4] are generalized using the recent new developments due to Maria L. Gandarias [8] and Nail H. Ibragimov [17, 19]. The main result is the new conserved vector (12). In particular, the results obtained here possibilite one to construct an infinite number of new conservation laws for equation (1). 
Acknowledgments. The comments and suggestions pointed out by the referee have improved the paper and the author would like to show his gratitude for the reviewer. It is also a pleasure to thank Professor Nail Ibragimov for sending to the author the manuscripts $[17,19]$. The author is grateful for FAPESP (Project No. 2011/19089-6).

\section{REFERENCES}

[1] M.A. Abdulwahhab, A.H. Bokhar, A.H. Kara and F.D. Zaman, On the Lie point symmetry analysis and solutions of the inviscid Burgers equation. Pramana J. Phys, 77 (2011), 407-414.

[2] Y. Bozhkov, I.L. Freire and N.H. Ibragimov, Group analysis of the Novikov equation, arXiv:1202.3954v1, (2012).

[3] M.S. Bruzón, M.L. Gandarias and N.H. Ibragimov, Self-adjoint sub-classes of generalized thin film equations. J. Math. Anal. Appl., 357 (2009), 307-313.

[4] I.L. Freire, Conservation laws for self-adjoint first order evolution equations. J. Nonlin. Math. Phys., 18(2) (2011), 279-290.

[5] I.L. Freire, Self-adjoint sub-classes of third and fourth-order evolution equations. Appl. Math. Comp., 217 (2011), 9467-9473.

[6] I.L. Freire and J.C.S. Sampaio, Nonlinear self-adjointness of a generalized fifthorder KdV equation. J. Phys. A: Math. Theor., 45 (2012), 032001.

[7] I.L. Freire, New classes of nonlinearly self-adjoint evolution equations of thirdand fifth-order, Commun. Nonlinear Sci. Num. Sci., 18 (2012), 493-499, DOI: 10.1016/j.cnsns.2012.08.022.

[8] M.L. Gandarias, Weak self-adjoint differential equations. J. Phys. A, 44 (2011), 262001 (6pp).

[9] M.L. Gandarias, Weak self-adjointness and conservation laws for a porous medium equation. Commun. Nonlinear Sci. Num. Sci., 17 (2012), 2342-2349.

[10] M.L. Gandarias, M. Redondo and M.S. Bruzón, Some weak self-adjoint HamiltonJacobi-Bellman equations arising in financial mathematics. Nonlin. Anal. RWA, 13 (2012), 340-347.

[11] M.L. Gandarias and M.S. Bruzón, Some conservation laws for a forced KdV equation. Nonlin. Anal. RWA, (2012), DOI: 10.1016/j.nonrwa.2012.03.013.

[12] N.H. Ibragimov, Integrating factors, adjoint equations and Lagrangians. J. Math. Anal. Appl., 318 (2006), 742-757. 
[13] N.H. Ibragimov, A new conservation theorem. J. Math. Anal. Appl., 333(1) (2007), 311-328.

[14] N.H. Ibragimov, Quasi-self-adjoint differential equations. Archives of ALGA, 3/4 (2007), 55-60.

[15] N.H. Ibragimov, M. Torrisi and R. Tracinà, Quasi self-adjoint nonlinear wave equations. J. Phys. A: Math. Theor., 43 (2010), 442001-442009.

[16] N.H. Ibragimov, M. Torrisi and R. Tracinà, Self-adjointness and conservation laws of a generalized Burgers equation. J. Phys. A: Math. Theor., 44 (2011), 145201-145206.

[17] N.H. Ibragimov, Nonlinear self-adjointness and conservation laws, arXiv: 1107.4877, (2011).

[18] N.H. Ibragimov, Nonlinear self-adjointness and conservation laws. J. Phys. A: Math. Theor., 44 (2011), 432002, 8 pp.

[19] N.H. Ibragimov, Nonlinear self-adjointness in constructing conservation laws. Archives of ALGA, 7/8 (2011), 1-90.

[20] N.H. Ibragimov, R.S. Khamitova and A. Valenti, Self-adjointness of a generalized Camassa-Holm equation. Appl. Math. Comp., 218 (2011), 2579-2583.

[21] M. Nadjafikhah, Lie symmetries of inviscid Burgers equation. Adv. Appl. Clifford Alg., 19(1) (2009), 101-112. 